\title{
Comparison of ETCO2 Value and Blood Gas PCO2 Value of Patients Receiving Non-invasive Mechanical Ventilation Treatment in Emergency Department
}

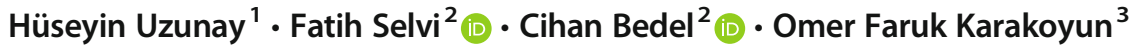

Accepted: 22 April 2021 / Published online: 27 April 2021

(C) The Author(s), under exclusive licence to Springer Nature Switzerland AG 2021

\begin{abstract}
Capnography is the non-invasive measurement and graphic representation of the partial pressure of $\mathrm{CO} 2$ in expiration. Although there are many studies in the literature comparing the partial pressure of carbon dioxide (pCO2) and end-tidal $\mathrm{CO} 2$ (ETCO2) values in patients who underwent IMV (invasive mechanical ventilation), there are no studies showing their interchangeable applicability in patients who received NIMV (non-IMV). We aimed to evaluate whether the use of ETCO2 in the treatment process can replace $\mathrm{pCO} 2$ use in patients scheduled for NIMV treatment in the emergency department. Patients who applied to the emergency department with respiratory distress between March 2019 and January 2020, who were diagnosed with acute cardiogenic edema or acute chronic obstructive pulmonary disease (COPD) exacerbation, and who needed NIMV were included in the study. General characteristics of the patients and the $\mathrm{pCO} 2$ and ETCO2 values were measured in the blood gas $1 \mathrm{~h}$ after the NIMV application was started. 64.2\% (99 patients) of the patients included in the study were male, and 35.8\% (55 patients) were female. The mean age of the patients included in the study was $69.1 \pm 12.2$ years. The mean $\mathrm{pCO} 2$ values were measured as 52.6 \pm 13.2 . The mean of ETCO2 values measured simultaneously was $33.6 \pm 10.1$. There was a significant difference between the controlled pCO2 values and ETCO2 values at the first hour of NIMV treatment (Z: $-10.640, p<0.001)$. The ETCO2 level was found to be different in our patients who received NIMV treatment, which could not be used instead of the pCO2 level.
\end{abstract}

Keywords Capnography $\cdot \mathrm{pCO} 2 \cdot \mathrm{ETCO} 2 \cdot$ Non-invasive mechanical ventilation

\section{Introduction}

When the complaints at admission to the emergency department are evaluated, the rate of dyspnea and respiratory distress is $2-3 \%$ [1]. Mechanical ventilation (MV) is a frequently used method in treating patients with respiratory distress because it reduces gas exchange and respiratory work and has two types

This article is part of the Topical Collection on Medicine

Cihan Bedel

cihanbedel@hotmail.com

1 Department of Emergency Medicine, Kas State Hospital, Antalya, Turkey

2 Department of Emergency Medicine, Health Science University, Antalya Training and Research Hospital, Antalya, Turkey

3 Department of Emergency Medicine, Mugla Sitki Kocman University Training Research Hospital, Mugla, Turkey of use: invasive mechanical ventilation (IMV) and noninvasive mechanical ventilation (NIMV) [2]. Blood gas analysis is an important indicator in the follow-up and treatment of patients, and especially the partial pressure ( $\mathrm{pCO} 2$ ) value of the carbon dioxide $(\mathrm{CO} 2)$ gas dissolved in the blood provides guidance for selecting the mode of the mechanical ventilator and adjusting its settings [3].

Capnography is the non-invasive measurement and graphic representation of the partial pressure of $\mathrm{CO} 2$ in expiration. It is also called end-tidal CO2 (ETCO2) because the measurement is taken from expiratory air. ETCO2 is mostly used in the emergency department to verify the location of the endotracheal tube in the intubated patient, to monitor the spontaneous return during cardiopulmonary resuscitation (CPR) in cardiac arrest cases, and to monitor the ventilation status in procedural sedoanalgesia. Using ETCO2 instead of pCO2 in the monitoring of intensive care patients undergoing IMV is an increasingly common method $[4,5]$.

It was found that there is a $1-4 \mathrm{mmHg}$ difference between the $\mathrm{pCO} 2$ value and the $\mathrm{ETCO} 2$ value in the use of 
capnography for the monitoring of intubated patients on a mechanical ventilator. However, it was found that the difference between pCO2 and ETCO2 increased in situations such as decreased lung perfusion, pulmonary embolism, dead space ventilation, and cardiac arrest [6]. Although there are many studies in the literature comparing pCO2 and $\mathrm{ETCO} 2$ values in patients who underwent IMV, there are no studies showing their interchangeable applicability in patients who received NIMV.

\section{Aims and Objectives}

Herein, we aim to describe to evaluate whether the use of ETCO2 in the treatment process can replace pCO2 use in patients scheduled for NIMV treatment in the emergency department.

\section{Methods}

\section{Study Participants}

This study was conducted as a prospective cross-sectional clinical study at a tertiary university hospital. Approval was obtained from the local ethics committee, and signed informed consent was obtained from all patients who participated in the study or from their first-degree relatives. Patients who applied to the emergency department with respiratory distress between March 2019 and January 2020, who were diagnosed with acute decompensated heart failure (ADHF) or acute chronic obstructive pulmonary disease (COPD) exacerbation, and who needed NIMV were included in the study. The need for NIMV was determined by clinical senior residents and specialist physicians. Being under 18 years of age, having pulmonary parenchymal diseases such as pneumonia, neuromuscular diseases, obstructive sleep apnea syndrome, obesity hypoventilation syndrome, patients who cannot tolerate NIMV treatment, and patients who did not agree to participate in the study were determined as the exclusion criteria. It was ensured that all patients who were treated were conscious, had sufficient cough and swallowing reflex, were clinically stable, and that there was no obstacle on the patient's face for the application of non-invasive ventilation.

Demographic information such as age, gender and history, vital signs (body temperature, pulse, blood pressure, respiratory rate), fingertip oxygen saturation ( $\mathrm{SpO} 2)$, main physical examination findings (rale, rhoncus, pretibial edema), and outcome conditions were noted in the patients who met the inclusion criteria. The pCO2 and ETCO 2 values were measured in the blood gas $1 \mathrm{~h}$ after the NIMV application was started.

After the physician decided to apply NIMV in treatment, treatments of patients started with a non-return mask using the continuous positive airway pressure (CPAP) and bilevel positive airway pressure (BiPAP) modes of the LTV 1200 (Vyaire Medical, Mettawa, Illinois) device. At the first hour of treatment, blood gas samples were taken to adjust the mode settings of the ventilator and to evaluate the treatment. Simultaneously, an A portable mainstream capnograph (Masimo EMMA Mainstream Capnograph; Masimo Corp., CA, USA) was placed between the connection of the mask and the hose, and ETCO2 was measured. Venous blood gas samples were taken from the patients with $22 \mathrm{G}$-tipped blood gas injectors. Testing of the samples taken were performed with the calibrated ABL800 FLEX (C) blood gas analyzer (ABL800) (Radiometer Medical ApS, Copenhagen, Denmark) device, and the $\mathrm{pCO} 2$ value was recorded. The hospitalization and intubation needs of the patients were obtained from the records on the hospital automation system.

\section{Primary Endpoint}

The primary endpoint of our study was the comparing pCO2 and ETCO2 values in patients who underwent NIMV.

\section{Secondary Endpoints}

The secondary aim is to compare the results of pCO2 and ETCO2 the suitability of using interchangeably.

\section{Statistical Analysis}

The data obtained were analyzed by using SPSS for Windows 22 package program. Descriptive numerical variables were presented as mean and standard deviation, and categorical variables were presented as number and percentage. Prior to the comparison of means, normal distribution was tested. The Mann-Whitney $U$ test was used to compare the means in nonnormally distributed groups, and the dependent groups' $t$-test was used to compare the means in normally distributed variables. Correlation analysis of variables was performed with the Pearson test for normally distributed data, and the Spearman test was used for non-normally distributed data. Bland-Altman analysis was used to determine the agreement between the two methods. Correlation coefficient $r$ value of $<$ 0.20 was considered very weak, $0.20-0.40$ weak, $0.40-0.60$ moderate, $0.60-0.80$ high, $>0.80$ very high correlation. $p<$ 0.05 was considered statistically significant.

\section{Results}

$64.2 \%$ (99 patients) of the patients included in the study were male, and $35.8 \%$ (55 patients) were female. The mean age of the patients included in the study was $69.1 \pm 12.2$ years. $55.1 \%$ ( 85 patients) of the pre-diagnoses made by emergency department physicians were COPD, and 44.9\% (69 patients) 
were ADHF. Twenty-nine (19\%) of the patients had no previously known diagnosis of COPD or ADHF and were evaluated as new diagnosis in the emergency department (newly diagnosed COPD, 4 patients; newly diagnosed ADHF, 25 patients). Physical examination findings were rale in 104 patients $(67.6 \%)$, rhoncus in 92 patients $(59.7 \%)$, and pretibial edema in 72 patients $(46.7 \%)$.

The mean pCO2 values were measured as $52.6 \pm 13.2$. The mean of ETCO2 values measured simultaneously was $33.6 \pm$ 10.1. There was a significant difference between the controlled pCO2 values and ETCO2 values at the first hour of NIMV treatment $(Z:-10.640, p<0.001)$. However, when the correlation of $\mathrm{pCO} 2$ and ETCO 2 values was examined, a moderate positive correlation was found $(p<0.001$, Spearman r: 0.569) (Table 1).

BiPAP mode was used in 107 (69.4\%) patients, and CPAP mode was used in $47(30.6 \%)$ patients in NIMV treatment. The mean systolic and diastolic blood pressure, respiratory

Table 1 Baseline characteristics of studied patients

\begin{tabular}{ll}
\hline Variables & Values $(n=154)$ \\
\hline Age (years) & \\
Mean \pm standard deviation & $69.1 \pm 12.2$ \\
Gender, $\mathrm{n}(\%)$ & \\
Male & $99(64.2)$ \\
Female & $55(35.8)$ \\
Vital signs & \\
SBP, mm Hg & $147.1 \pm 37.3$ \\
DBP, mm Hg & $85.3 \pm 9.4$ \\
Heart rate, beats/min & $102.3 \pm 22.3$ \\
Body temperature & $36.3 \pm 0.2$ \\
Pre-diagnoses, $\mathrm{n}(\%)$ & \\
Chronic obstructive pulmonary disease & $85(55.1)$ \\
Acute Decompensated Heart Failure & $69(44.9)$ \\
Clinical findings, $\mathrm{n}(\%)$ & $104(67.6)$ \\
Ralles & $92(59.7)$ \\
Ronchus & $72(46.7)$ \\
Pretibal edema & \\
Previous history , $\mathrm{n}(\%)$ & $52.6 \pm 13.2$ \\
Hypertension & $33.6 \pm 10.1$ \\
Diabetes mellitus & \\
Others & $25(16.2)$ \\
1st hour analysis & $48(31.2)$ \\
pCO2 & $11(7.1)$ \\
ETCO2 & \\
Outcome & \\
ICU admission & \\
\hline
\end{tabular}

$S B P$ systolic blood pressure, $D B P$ diastolic blood pressure, $P C O 2$ partial pressure of carbon dioxide, ETCO2 end-tidal carbon dioxide, $I C U$ intensive care unit rate, and $\mathrm{O} 2$ saturation levels were found to be significantly higher in CPAP mode usage group compared to the BiPAP mode usage group ( $p<0.05$ for all markers). The mean pCO2 and ETCO2 values were found to be significantly higher in BiPAP mode usage group compared to the CPAP mode usage group ( $p<0.001$ ) (Table 2). Scatter plot of ETCO2 vs. pCO2 levels is shown in Fig. 1. Bland-Altman plot illustrating the agreement between pCO2 and ETCO2 is shown in Fig. 2. It was observed that $59.1 \%$ (91 patients) of the patients included in the study were hospitalized according to their outcomes. $35.7 \%$ (55 patients) of the patients who were hospitalized were admitted to the intensive care unit. $25.4 \%$ (14 patients) of the patients who were taken into the intensive care unit resulted in endotracheal intubation.

\section{Discussion}

ETCO2 monitoring with capnometer is a method routinely used in intensive care units to evaluate the respiratory state and rate in patients receiving IMV treatment, and it is advantageous as it does not require intervention. While the use of invasive ETCO2 has become widespread by clinicians who monitor patients in intensive care units, it has been thought that $\mathrm{CO} 2$ in physiological dead spaces may reflect the $\mathrm{CO} 2$ pressure in the blood. In the literature, the relationship between ETCO2 and pCO2 was first shown with 31 patients in 2000 [7]. In the literature, there are many studies evaluating the usability of ETCO2 instead of pCO2 in blood gas of patients undergoing IMV treatment [8, 9]. In 2017, in a study by Nassar et al., correlation coefficients between pCO2 and ETCO2 values were found to be between 0.6 and 0.9 [10].

A recent study investigating simultaneous ETCO2 and pCO2 measurements in blood gas in SIMV (synchronized intermittent mandatory ventilation), CPAP, and T-tube modes of IMV therapy demonstrated a moderate correlation between measured pCO2 and ETCO2 values. ${ }^{9}$ In 2013, Singh et al. conducted a study in the neonatal intensive care unit and found a moderate correlation between the ETCO2 level and pCO2 level in patients treated with IMV [11]. In a study by Fujimoto et al. conducted in 2019 with 30 patients with respiratory failure and 18 patients with hypercapnia, a significant difference was found between ETCO2 and pCO2 [12].

In 2017, Schwarz et al. evaluated 3 measurements of pCO2 values at the beginning, 30th minute and 60th minute with the Sidestream ETCO2 measurements taken by cannula from the endotracheal tube of patients under IMV treatment in the Bland-Altman analysis. The mean $\mathrm{pCO} 2$ values of all patients were found to be $42.4 \pm 8.6 \mathrm{mmHg}$, and the mean of ETCO2 values were $36 \pm 7.5 \mathrm{mmHg}$. A significant correlation was found between the $\mathrm{pCO} 2$ value and $\mathrm{ETCO} 2$ value. In addition, it was emphasized that ETCO2 was so inconsistent that it could not be used instead of pCO2 [13]. In our study, the mean 
Table 2 Baseline characteristics of treatment options

\begin{tabular}{llll}
\hline Variables & CPAP $(n=47)$ & BIPAP $(n=107)$ & $p$ \\
\hline Age (year) & $71.66 \pm 12.45$ & $67.84 \pm 11.84$ & 0.061 \\
Sex (male/female) & $30 / 17$ & $69 / 38$ & 0.539 \\
Mean systolic blood pressure & $163.74 \pm 41.75$ & $140.18 \pm 32.59$ & 0.002 \\
Mean diastolic blood pressure & $97.45 \pm 27.81$ & $82.84 \pm 16.68$ & 0.002 \\
Mean respiratory rate & $30.23 \pm 7.29$ & $26.95 \pm 7.61$ & 0.014 \\
Mean O2 saturation $(\%)$ & $88.43 \pm 7.41$ & $84.68 \pm 10.12$ & $<0.001$ \\
Mean pulse/min & $106.3 \pm 21.71$ & $100.11 \pm 22.59$ & 0.180 \\
ETCO2 levels & $31.02 \pm 9.28$ & $34.81 \pm 10.17$ & $<0.001$ \\
PaCO2 levels & $45.91 \pm 10.52$ & $55.54 \pm 13.44$ & 0.031 \\
\hline
\end{tabular}

pCO2 value was measured as $52.6 \pm 13.2 \mathrm{mmHg}$ in the blood gas sample taken at the first hour of NIMV treatment. The mean of ETCO2 values measured simultaneously was 33.6 $\pm 10.1 \mathrm{mmHg}$. The reason why the $\mathrm{pCO} 2$ value is higher than the literature may be that the study was conducted in the emergency department and the patients included in our study were evaluated at the first treatment without providing respiratory and metabolic stabilization.

In our study, similar to the literature, a significant difference was found between the ETCO 2 measurement and $\mathrm{pCO} 2$ level $(\mathrm{Z}:-10.640, p<0.001)$. For this reason, we think that it is not appropriate to use the ETCO2 value numerically instead of pCO2 level. Similar to the literature, the results of our study showed a moderate positive correlation between the $\mathrm{pCO} 2$ value and ETCO2 value ( $p<0.001, \mathrm{r}: 0.569)$. In the literature we reviewed, all the publications in which the ETCO2 level was measured consisted of patients who were intubated or received IMV treatment in intensive care units. We think that the correlation coefficient between the ETCO2 measurement and pCO2 level was lower due to the fact that our patient group consisted of patients receiving NIMV treatment in the emergency department. The reason for this may be that the

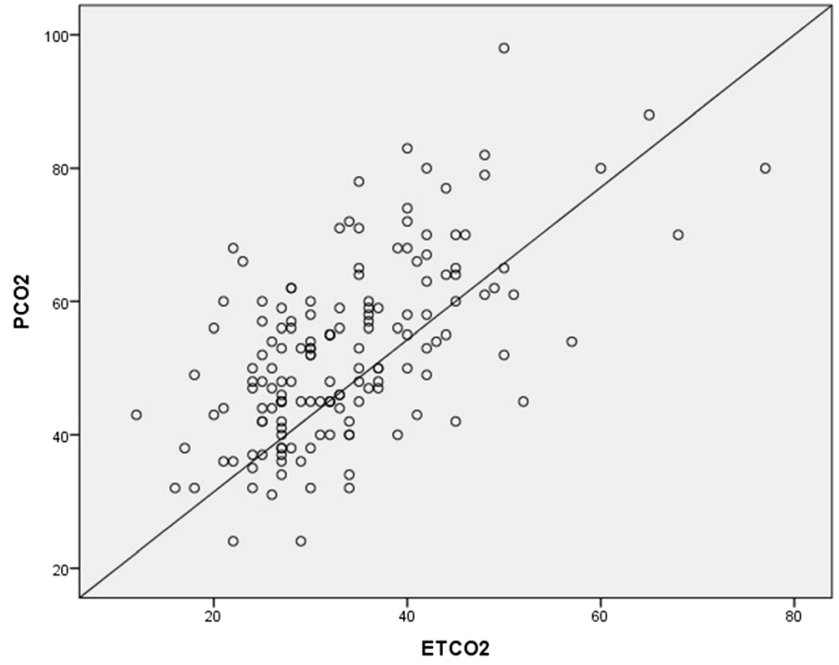

Fig. 1. Scatter plot of $\mathrm{ETCO} 2$ versus $\mathrm{pCO} 2$. non-functioning dead space area is more in NIMV applied patients, that there is an uncontrolled air leak outside the system, or that the device sensor used for mainstream measurement is fogged.

ETCO2 is mostly recommended to be used in patients receiving IMV treatment under anesthesia and in the intensive care unit. Despite its use under these conditions, it has been found in studies in the literature that the use of ETCO2 instead of pCO2 value is limited [14]. In our study, in addition to the literature, we think that the ETCO2 level can be used to evaluate the treatment monitoring in patients receiving NIMV treatment.

This study has certain limitations. The first of these was that the first hour observation time was quite short. Another limitation of our study was that although our study was designed prospectively, it was conducted with a relatively small number of patients. Furthermore, the single-center design of our study increased bias. Another limitation was the fact that the NIMV decision was not made by the same physicians may have influenced the results. In addition, the lack of evaluation of the time from the onset of symptoms to hospital admission may have affected the results.

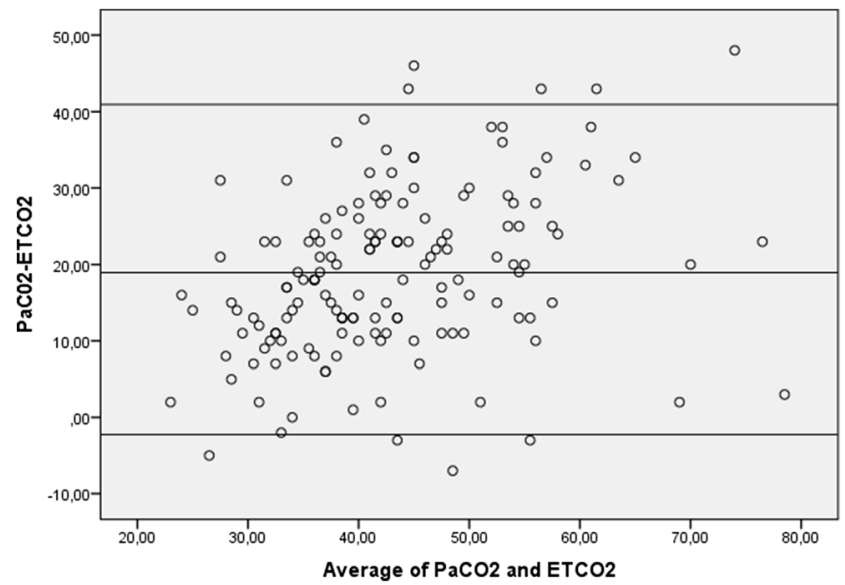

Fig. 2. Bland-Altman plot illustrating the agreement between ETCO2 and $\mathrm{pCO} 2$ 


\section{Conclusion}

The ETCO2 level was found to be different in our patients who received NIMV treatment, which could not be used instead of the $\mathrm{pCO} 2$ level. However, the positive moderate correlation between the ETCO2 level and $\mathrm{pCO} 2$ level and the monitoring of NIMV treatment with the ETCO2 level may have benefits such as predicting an unexpected increase in pCO2 levels or making the decision to intubate patients faster.

Author Contribution HU, FS, and OFK conceptualized the idea. HU, FS, and $\mathrm{CB}$ reviewed the literature and collected the data with the help of FS and $\mathrm{CB}$ formulated the tables. $\mathrm{HU}, \mathrm{FS}, \mathrm{CB}$, and OFK wrote the main draft of the manuscript. HU and FS supervised the project.

Data Availability Database is available upon request.

Code Availability Not applicable

\section{Declarations}

Ethics approval This study was approved by the ethics committee of the Antalya Training and research hospital. All procedures performed in studies involving human participants were in accordance with the ethical standards of the institutional and/or national research committee and with the 1964 Helsinki Declaration and its later amendments or comparable ethical standards.

Consent to Participate Informed consent is applicable for this study.

Consent for Publication Not applicable

Conflict of Interest The authors declare that they have no conflict of interest.

\section{References}

1. Ray P, Birolleau S, Lefort Y, Becquemin MH, Beigelman C, Isnard $\mathrm{R}$, et al. Acute respiratory failure in the elderly: etiology, emergency diagnosis and prognosis. Crit Care. 2006;10(3):R82.

2. Fichtner F, Moerer O, Weber-Carstens S, Nothacker M, Kaisers U, Laudi $\mathrm{S}$, et al. Clinical guideline for treating acute respiratory insufficiency with invasive ventilation and extracorporeal membrane oxygenation: evidence-based recommendations for choosing modes and setting parameters of mechanical ventilation. Respiration. 2019;98(4):357-72.

3. Al Ashry HS, Richards JB, Fisher DF, Sankoff J, Seigel TA, Angotti LB, et al. Emergency department blood gas utilization and changes in ventilator settings. Respir Care. 2018;63(1):36-42.

4. Long B, Koyfman A, Vivirito MA. Capnography in the emergency department: a review of uses, waveforms, and limitations. J Emerg Med. 2017;53(6):829-42.

5. Sereceda-Sánchez FJ, Molina-Mula J. Capnography as instrument for airway management during basic instrumental CPR manoeuvers. Resuscitation. 2019;138:132-3. https://doi.org/10. 1016/j.resuscitation.2019.03.007.

6. Walsh BK, Crotwell DN, Restrepo RD. Capnography/capnometry during mechanical ventilation: 2011. Respir Care. 2011;56(4):5039.

7. Fletcher R, Boris-Moller F. Can we improve the estimate of arterial PCO2 from end tidal PCO2? Eur J Anaesthesiol. 2000;17(5):30610.

8. McSwain SD, Hamel DS, Smith PB, Gentile MA, Srinivasan S, Meliones JN, et al. End tidal and arterial carbon dioxide measurements correlate across all levels of physiologic dead space. Respir Care. 2010;55(3):288-93.

9. Razi E, Moosavi GA, Omidi K, Khakpour Saebi A, Razi A. Correlation of end tidal carbon dioxide with arterial carbon dioxide in mechanically ventilated patients. Arch Trauma Res. 2012;1(2): $58-62$.

10. Nassar BS, Schmidt GA. Estimating arterial partial pressure of carbon dioxide in ventilated patients: how valid are surrogate measures? Ann Am Thorac Soc. 2017;14(6):1005-14.

11. Singh BS, Gilbert U, Singh S, Govindaswami B. Sidestream microstream end tidal carbon dioxide measurements and blood gas correlations in neonatal intensive care unit. Pediatr Pulmonol. 2013;48(3):250-6.

12. Fujimoto S, Suzuki M, Sakamoto K, Ibusuki R, Tamura K, Shiozawa A, et al. Comparison of end tidal, arterial, venous, and transcutaneous PCO2. Respir Care. 2019;64(10):1208-14.

13. Schwarz SB, Windisch W, Magnet FS, Schmoor C, Karagiannidis $\mathrm{C}$, Callegari J, et al. Continuous non invasive PCO2 monitoring in weaning patients: transcutaneous is advantageous over end tidal PCO2. Respirology. 2017;22(8):1579-84.

14. Magnet FS, Windisch W, Storre JH. Monitoring of pCO2 during ventilation. Med Klin Intensivmed Notfallmed. 2016;111(3):2027.

Publisher's Note Springer Nature remains neutral with regard to jurisdictional claims in published maps and institutional affiliations. 